\title{
Application of Instantaneous Parameter Characteristic in Active Lamb Wave Based Monitoring of Plate Structural Health
}

\author{
Baochun $\mathrm{Xu}^{1,2, *}$, Mulan Wang ${ }^{2, *}$, Peijuan Li ${ }^{1}$, Qihua Cheng ${ }^{1}$ and Yunlong Sheng ${ }^{1}$ \\ 1 Nanjing Institute of Technology, Nanjing 211167, China; lpj4444@126.com (P.L.); chenqh@njit.edu.cn (Q.C.); \\ ShengYL@njit.edu.cn (Y.S.) \\ 2 Jiangsu Key Laboratory of Advanced Numerical Control Technology, Nanjing 211167, China \\ * Correspondence: baochun_xu@njit.edu.cn (B.X.); wangml@njit.edu.cn (M.W.); Tel.: +86-25-8611-8583 (B.X.)
}

Received: 18 July 2020; Accepted: 12 August 2020; Published: 14 August 2020

\begin{abstract}
In the active Lamb wave based monitoring of plate structural health, it is difficult to extract damage information from active Lamb waves based on single sensor collection. Based on the Hilbert-Huang transform (HHT) instantaneous processing method, this paper proposes to study the damage information carried by a single sensor monitoring signal from the instantaneous parameter characteristics of the signal. The instantaneous phase change caused by the phase difference between the damage scattering wave and the direct wave is studied. The change of the marginal spectrum amplitude in the effective range caused by the damage scattering wave is studied in continuous multiple frequency bands. Finally, the damage information extraction based on a single sensor monitoring signal is realized. From the model analysis and experimental results, it is reliable and feasible to realize the active Lamb wave based monitoring of plate structural health according to the instantaneous parameter change characteristics from a single sensor signal.
\end{abstract}

Keywords: plate structural health; active Lamb wave; HHT; instantaneous phase; marginal spectrum

\section{Introduction}

Modern aircraft mostly adopt composite plate structures to meet the requirements for multifunctionality, high mobility, and high reliability. However, during service, damage to the composite plate structures is unavoidable, which may abruptly reduce the strength and reliability of the plate structures and pose serious threats to aircraft safety. At present, there are two groups of methods for plate structural health monitoring (SHM): active and passive monitoring methods [1,2]. Passive SHM methods only "listen" to the structure but do not interact with it. Acoustic emission (AE) is a typical passive SHM method that has been applied to the monitoring of plate damage [3-5]. However, due to a variety of factors, such as damage suddenness, damage complexity, and signal nonreproducibility, the requirements for monitoring equipment energy, signal processing methods, and sensor placement are high [6,7]. Therefore, the application of passive monitoring methods is limited. On the other hand, active SHM methods use sensors to analyze the structure to detect damage. Active monitoring methods use actuators to excite the structure and sensors to receive the structural response signals needed for structural health monitoring. Besides, active monitoring methods are flexible, the structure response is controllable, and the sensing signal has good repeatability. Active Lamb wave technology is widely used in the health monitoring of plate structures [8-10].

A Lamb wave is an elastic wave whose propagation distance is relatively long and amplitude loss in a thin plate structure is small. Therefore, by using several sensors in combination with Lamb waves, it could be possible to achieve large-scale plate area monitoring. An active Lamb wave based 
monitoring of a plate structural health generates the Lamb wave by using an actuator and excites a plate structure. Then, the response signal is received by a sensor. Finally, the health information is extracted by signal processing to realize the SHM of a plate. A Lamb wave produced during the active Lamb wave based monitoring is called an active Lamb wave. Although Lamb waves have multimode and dispersion characteristics, an appropriate excitation signal and center frequency should be selected for active monitoring to control the Lamb wave waveform and mode of the plate response [8]. Accordingly, active Lamb wave based monitoring has become a research hotspot [11-13]. In active Lamb wave based plate structural health monitoring, the array sensor, which has strong anti-interference, is used to acquire enough phase information of the Lamb wave in the plate to realize the plate structure health evaluation $[14,15]$. However, array sensor monitoring has directionality, and thus there are monitoring blind spots and it is difficult to arrange in the narrow space of the plate structure. There are also monitoring methods to extract damage information based on a single sensing signal. The smallest unit of these monitoring methods only needs one exciter and one sensor. It can not only form a network of multiple units, realize large area monitoring, and eliminate blind spots but also arrange a monitoring unit in a narrow space. The sensor layout is flexible. The classic method of extracting damage information by this monitoring method is based on the difference of a single sensing signal before and after damage [16]. This method of extracting damage information is intuitionistic, but its anti-interference is poor. Therefore, it is difficult to keep the starting time of each acquisition consistent, and thus there is a phase difference between the collected data. In this case, even if the plate structure is undamaged, the difference between the signals is like the scattering signals, which makes it easy to cause misjudgment. Moreover, a Lamb wave parameter model has been established in the time domain to analyze the change of the sensor signal parameter vector, which realizes the damage evaluation of aluminum plate [17]. However, this method is related to plate structure and material, and thus it has poor universality. There are advantages to studying signal characteristic change from a frequency domain, such as anti-interference and correlation between characteristic change and frequency. However, there have been few reports on extracting damage information directly from the frequency domain of single sensing data because the Lamb wave carrying damage information is a typical nonstationary signal, and signal processing based on Fourier transform cannot truly reflect the change characteristics of a damage signal. However, time-frequency analysis methods, such as Wigner Ville distribution, short-time Fourier transform, and wavelet transform, have limitations in theory and make it difficult to support in-depth analysis of the changes of the active Lamb wave monitoring signal $[18,19]$. Therefore, in the research of active Lamb wave monitoring technology, finding an advanced signal processing method to extract damage information directly from a single sensing signal has been a hot spot of structural health monitoring research.

Hilbert-Huang transform (HHT) represents a signal processing method that uses a signal extreme point to decompose the signal adaptively and then analyzes instantaneous signal characteristics using Hilbert transform (HT) [20]. Moreover, it is a common method for nonstationary signal processing. Law et al. [18] stated that HHT has been a promising technique for extracting the properties of nonlinear and nonstationary signals. They used HHT to process the AE data of a spindle box and found that the marginal spectrum of acoustic emission had a better correspondence with the variation of spindle load, bearing, and base geometry with the temperature. Nalband et al. [19] suggested using the smoothed pseudo-Wigner-Ville distribution (SPWVD) and HHT for the analysis of body joint motion vibration signals. The time-frequency representation of the suggested methods was considered as a time-frequency image from which the statistical features based on the image pixels were extracted. The results showed that the highest classification accuracy of $88.76 \%$ was obtained when the features were extracted by the HHT. Liu et al. [21] used HHT to process the sensing signals emitted by the mechanical looseness signals. The loosening signal with strong background noise was first decomposed by empirical mode decomposition (EMD), and then the instantaneous frequency of the first intrinsic mode function (IMF) was calculated to accurately determine the loose position. Chen et al. [22] believed that HHT was a new method for time-frequency analysis. They used HHT to 
determine the variation in the normalized instantaneous frequency as an index vector of composite wing damage. While studying the simulated vibration signal of a composite wing, even when the effect of noise was considered, the index vector could still detect small damage ( $0.03 \%$ of the entire wing area) that caused a decrease in the elastic parameters. Biswas et al. [23] compared the inherent strengths and weaknesses of CWT and HHT in quantifying the scale-and location-specific soil spatial variation. The HHT method showed superior performance in identifying the scales and locations of soil spatial variability over the CWT because HHT used a more flexible basis for interpreting real data and could deal with nonlinear problems. Susanto et al. [24] applied HHT to analyze and process milling signals. After the signals were decomposed into IMFs by EMD, it was found that each one of them contained a unique vibration mode caused by machining, and thus every cutting phenomenon was detected easily in the Hilbert spectrum. Xu et al. [25] introduced HHT signal processing technology into the active Lamb wave based monitoring of plate structural health. In their method, the marginal spectrum of the monitoring signal was obtained and compared with the spectrum. It was concluded that the sensitivity of the marginal spectrum to signal variation is higher than that of the spectrum. According to the above-mentioned literature, HHT performed well in the information processing of nonstationary signals. Namely, HHT could adaptively decompose a signal into a few components and process them by HT. The signal could be observed from the aspect of time-domain intrinsic sequence, time-frequency variation, and energy distribution, and thus the signal information could be described in more detail.

Based on the previous two paragraphs, the following conclusions can be drawn: A Lamb wave is a typical nonstationary signal sensitive to structural damage, and HHT can be applied to nonstationary signal processing to extract the abnormal information that cannot be extracted by the conventional signal processing. The active monitoring method in this paper is based on the superposition of a damage scattering wave in the monitoring signal. There is a lack of research on the change of the monitoring signal caused by the scattering wave in the frequency domain or frequency-time domain. Compared with the signal characteristics of abnormal events in prior work [18,19,21-24], in the active monitoring method in this paper, the scattering signal frequency is not only consistent with the frequency of monitoring signal but also far less than the energy of monitoring. Therefore, it is worth studying what changes the scattering signal causes in the monitoring signal. Some papers have carried out preliminary research on this; for example, one study [25] directly used a marginal spectrum to process monitoring signals and found that a marginal spectrum is more sensitive to a change of monitoring signals than spectrum. However, these papers did not explain the relationship between the scattering wave and the instantaneous parameter change of the monitoring signal nor did they answer why the EMD decomposition results of the same state monitoring signals were quite different. Thus, they could not be used to study changes before and after the damage. In this paper, the relationship between the scattering signal and the change in the signal instantaneous phase is studied, and the consistency of the signal EMD decomposition is solved, which makes it feasible to use the change in the instantaneous parameter characteristics of a single sensing signal to realize the active Lamb wave based monitoring of the plate structural health.

The rest of this paper is organized as follows: In Section 2, HHT-based signal processing and the principle of active Lamb wave based monitoring of a plate structural health is presented. In Section 3, the monitoring signal model is established, and the relationship between the attenuation coefficient and superposition of the scattering wave and instantaneous phase change of the active Lamb wave is discussed. In Section 4 , the change in the active Lamb wave marginal spectrum in the frequency domain caused by plate structure damage is analyzed. In Section 5, the relationship between the instantaneous phase change and the marginal spectrum change of a monitoring signal is discussed. Lastly, conclusions are drawn in Section 6. 


\section{HHT-Based Signal Processing and Active Lamb Wave Based Monitoring Method}

\subsection{HHT-Based Signal Processing}

Following the demand of HT for the signal condition, HHT assumes that a signal is composed of several IMFs, each of which can be subjected to HT. However, in practice, most signals do not conform to the IMF definition. With HHT, a signal can be decomposed into a few IMFs by EMD, and then HT can be used to perform signal analysis in the time-frequency domain. The time-frequency processing procedure is given below.

Assume that the $k$ th-order IMF expression of a signal is $c_{k}(t)$, then, according to HHT theory, $c_{k}(t)$ can be defined as $c_{k}(t)=A_{k}(t) \cos \left(\phi_{k}(t)\right)$, where $A_{k}(t)>0$. Let the HT of $c_{k}(t)$ be $\hat{c_{k}}(t)$, and then the analytical expression of the $k$ th-order IMF can be written as [20]

$$
Z_{k}(t)=c_{k}(t)+j \hat{c_{k}}(t)=A_{k} \cos \left(\phi_{k}(t)\right)+j A_{k}(t) \sin \left(\phi_{k}(t)\right)=A_{k}(t) e^{j \phi_{k}(t)}
$$

Next, the instantaneous phase is given by

$$
\phi_{\mathrm{k}}(\mathrm{t})=\arctan \left(\frac{\hat{\hat{\mathrm{k}}_{\mathrm{k}}}(\mathrm{t})}{\mathrm{c}_{\mathrm{k}}(\mathrm{t})}\right)
$$

The instantaneous frequency $\left(\mathrm{f}_{\mathrm{i}}(\mathrm{t})\right)$ is defined as a derivative of the instantaneous phase $(\phi(t))$, and the $f_{i}(t)$ of Equation (2) is given by

$$
f_{i k}(t)=1 / 2 \pi \times d\left(\phi_{k}(t)\right) / d t
$$

Then, Equation (1) can be written as [20]

$$
Z_{k}(t)=A_{k}(t) e^{j \int 2 \pi f_{i k}(t) d t}
$$

where $\left|Z_{k}(t)\right|$ represents the amplitude of a Hilbert spectrum, that is, $A_{k}(t)$. Thus, $\left(t, f_{i k}, A_{k}\right)$ is the Hilbert spectrum of the $k^{\text {th }}$-order IMF, where each point $\left(t, f_{i k}\right)$ in Equation (4) corresponds to $A_{k}$. If this relationship is defined as $\mathrm{H}_{\mathrm{k}}\left(\mathrm{t}, \mathrm{f}_{\mathrm{ik}}\right)$, then the Hilbert spectrum of all the IMFs of a signal can be defined as $\mathrm{H}\left(\mathrm{t}, \mathrm{f}_{\mathrm{i}}\right)$, and the marginal spectrum in the HHT can be expressed as [20]

$$
h(f)=\int H\left(t, f_{i k}\right) d t
$$

The above processing method includes the instantaneous phase, instantaneous frequency, Hilbert spectrum, and marginal spectrum. More detailed descriptions of Equations (1)-(5) can be found in prior work [20]. The definition of instantaneous frequency can be regarded as a general extension of the frequency definition. In this paper, the instantaneous parameter characteristics processing technique of HHT is used to explore the active Lamb wave based monitoring signal, and changes in the instantaneous parameter characteristics before and after damage are analyzed.

\subsection{Active Lamb Wave Based Monitoring Method}

Various damages inside a structure increase the stress concentration and crack probability, and damage boundaries cause scattering of the propagation waves in a structure. Thus, a damage scattering wave can be considered as a wave that comes from another signal source. The active Lamb wave based monitoring of plate structural health is based on this principle. In the active monitoring of a plate structure, if a waveform excited by the actuator encounters damage on its propagation path, then scattering occurs. In that case, the signal received by the sensor is composed of the scattering wave and direct wave, as shown in Figure 1a. It should be noted that the damage location is not on the straight line with the actuator and sensor as two points. In practice, as for a secondary 
wave source, the amplitude of a damage scattering wave attenuates significantly, and no change in the waveform can be observed after the superposition with the direct wave. The active Lamb wave based monitoring system adopted in this paper was developed by the Structural Health Monitoring and Forecasting Group of the State Key Laboratory of Mechanics and Control of Mechanical Structures at Nanjing University of Aeronautics and Astronautics (NUAA). It includes the wave excitation module, the switch control module, the charge amplifier module, and the data acquisition module, as shown in Figure $1 \mathrm{~b}$. The actuators and sensors are both piezoelectric patches (PZTs) [26]. The excitation signal of the actuators is a tone burst signal, and its mathematical expression is given by

$$
\mathrm{u}(\mathrm{t})=\mathrm{A} \times\left[\mathrm{H}(\mathrm{t})-\mathrm{H}\left(\mathrm{t}-\mathrm{n} / \mathrm{f}_{\mathrm{c}}\right)\right] \times\left(1-\cos 2 \pi \mathrm{f}_{\mathrm{c}} \mathrm{t} / \mathrm{n}\right) \times \sin 2 \pi \mathrm{f}_{\mathrm{c}} \mathrm{t}
$$

where A represents the signal amplitude modulation, $\mathrm{f}_{\mathrm{c}}$ denotes the center frequency, $\mathrm{n}$ is the number of signal wave peaks, and $\mathrm{H}(\mathrm{t})$ denotes the Heaviside step function [8].

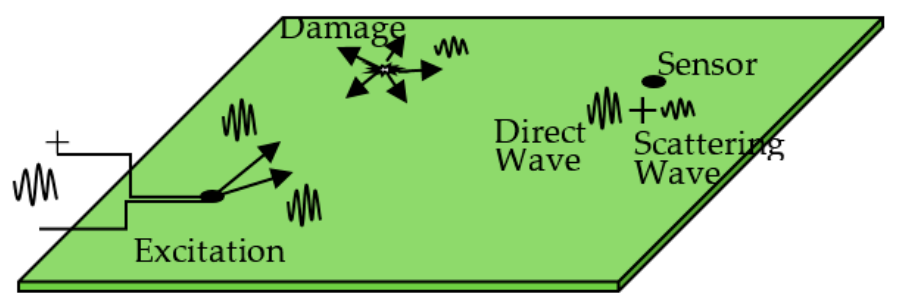

(a)

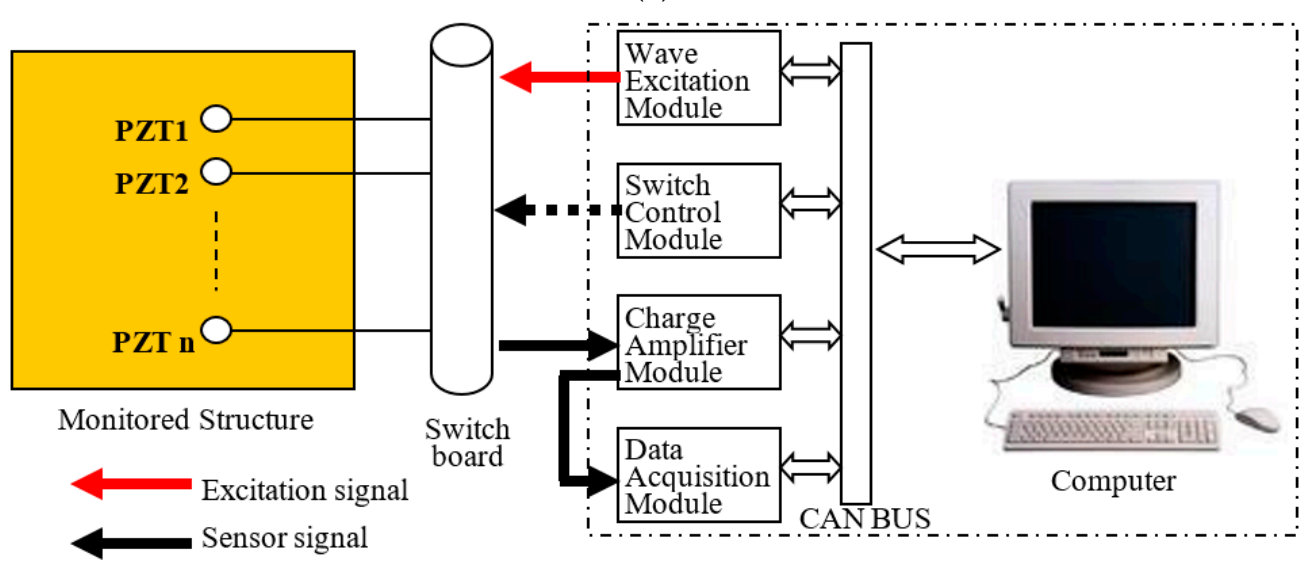

(b)

Figure 1. The active monitoring principle and the active monitoring system. (a) Schematic diagram of the active monitoring principle of a plate structure; (b) Structural diagram of an active Lamb wave based monitoring system.

The experimental system used for the active Lamb wave based monitoring of the plate structure is presented in Figure 2. The excitation signal had a center frequency of $150 \mathrm{kHz}$ and five peaks as shown in Figure 2a. As shown in Figure 2b, there were three wave packets in the plate response. Wave packet 1 occurred at the same time as the excitation signal, and it apparently denoted crosstalk. Thus, it could be neglected. Wave packet 2 represented the propagation wave of the excitation signal in the plate structure. Wave packet 3 could also be ignored because it had relatively low energy and was mainly composed of the signals reflected by the plate structure edges. Therefore, Wave packet 2 was considered to be a response of the plate structure to the excitation signal, and it was easily identifiable in the sensing signal. The wavelet transform of the signals is shown in Figure $2 \mathrm{c}, \mathrm{d}$, wherein it can be seen that Wave packet 2 almost maintained a consistent center frequency with the excitation signal. A tone burst signal was adopted as the excitation signal, mainly because of the high identification degree of its response in the plate as well as its simple waveform structure. 
Furthermore, the center frequency of the excitation signal could also be regulated to acquire a relatively ideal response wave in the plate structure, which not only facilitated the extraction of the signal time-frequency parameters but also reduced the interferences of parameter comparisons before and after damage. In this study, Wave packet 2 in Figure $2 b$ was taken as the research object, and the HHT time-frequency processing technology was used to analyze its instantaneous phase and marginal spectrum before and after damage.

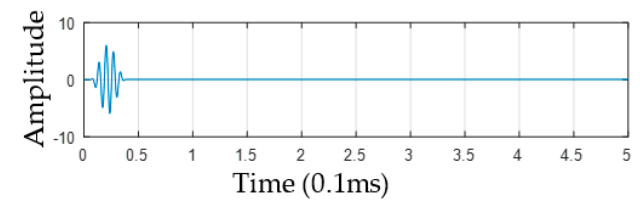

(a)

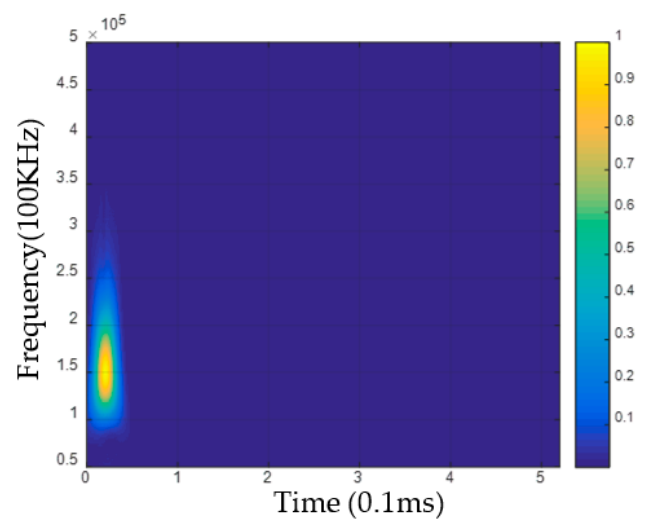

(c)

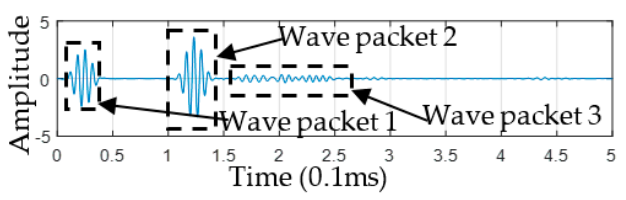

(b)

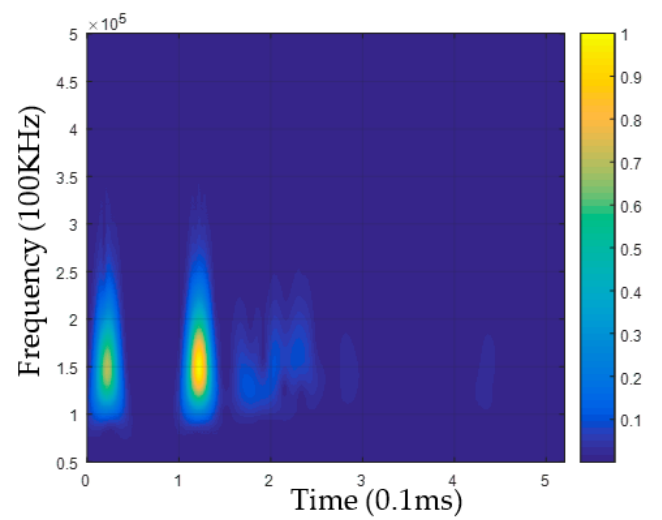

(d)

Figure 2. Lamb wave excited in the plate structure and the associated wavelet transform. (a) Excitation signal; (b) Plate structure response signal; (c) Time-frequency image of the wavelet transform of the excitation signal; (d) Time-frequency image of the wavelet transform of the plate structure response signal.

\section{Study on Instantaneous Phase of an Active Lamb Wave Monitoring Signal}

The location and size of the damage in the plate structure monitoring area are unpredictable. It is necessary to establish a signal model to study the change of the signal instantaneous phase under different damage conditions and reveal the relationship between them to provide the basis for the analysis conclusion of the actual experimental data.

\subsection{Establishment of the Monitoring Signal Model}

The active Lamb wave based monitoring of a plate structure utilizes the excitation source to generate a Lamb wave in a plate structure, and the Lamb wave scatters when it encounters the damage. In this way, a Lamb wave that is arriving directly at a sensor is superposed with the scattering wave, and the sensing signal contains the information on the plate structure health. This sensing signal is called a monitoring signal, and it can be defined as the sum of a direct wave and a scattering wave. A suitable monitoring signal model is needed for the following simulation experiment. According to the characteristics of the active Lamb wave based monitoring technology and the experimental data, a direct wave and a scattering wave were constructed to establish an approximate monitoring signal model.

As introduced in Section 2.2, the tone burst signal, which is used as an excitation signal, generates a Lamb wave that propagates in the plate and has a waveform that is almost consistent with the excitation signal (see Wave packet 2 in Figure 2b). Therefore, a tone burst signal (see Equation (6)) was used as a direct wave in the monitoring signal model. The scattering wave is the scattering of the Lamb wave 
in propagating caused by damage. This means that the scattering wave and the direct wave come from the same excitation source, and their waveforms are similar. The monitoring signal model was defined as the sum of the direct wave and the scattering wave. The direct wave remains unchanged before and after the damage, and thus the scattering signal is the dominant factor of the signal model. That is to say, the expression of the signal model is determined by the waveform and superposition location of the scattering wave. The damage conditions, such as the location and size, directly affect the waveform and superposition location of the scattering waves, and it is impractical to analyze the instantaneous phase change of the damage signal model directly according to the actual damage conditions. This requires a reasonable design so that the experimental process not only simulates the actual situation but also the instantaneous phase change analysis is feasible.

For the monitoring signal, the influence of the damage condition on the scattering wave is ultimately reflected in the waveform and superposition location. Generally, the larger the damage size is, the higher the energy of the scattering wave is; the longer the propagation path of the scattering wave is, the more energy the scattering wave consumes. Other factors, such as scattering angle and plate material, also affect the energy of scattering waves [27-29]. However, these factors will not change the center frequency of the scattering wave, and the scattering wave and the direct wave come from the same excitation source. Thus, the attenuation of the scattering wave energy can be approximately the attenuation of the wave amplitude. Therefore, the attenuation coefficient $\alpha$ of the scattering signal is simplified as the ratio of the amplitude of the scattering wave and the direct wave. In the damage signal model, the damage scattering wave is the product of direct wave and attenuation coefficient $\alpha$. By changing $\alpha$, the signal model can simulate the scattering waves with different energy.

The propagation distance of the direct wave is constant before and after the damage, and thus the superposition site is determined by the propagation distance of the damage scattering wave, in other words, by the location of the damage. The three positions of the damage, excitation, and sensor form a triangle, and the propagation distance of damage scattering signal is larger than that of the direct wave. Therefore, the scattering wave expression shifted to the right $\Delta t$ on the time axis and then superposes to the direct wave. With different $\Delta t$ in the simulation experiment, the signal model can simulate the different damage locations.

According to the above analysis, the mathematical expression of the scattering wave is given by

$$
\mathrm{u}^{\prime}(\mathrm{t})=\alpha \mathrm{u}(\mathrm{t}+\Delta \mathrm{t})=\alpha \times \mathrm{A} \times\left[\mathrm{H}(\mathrm{t}+\Delta \mathrm{t})-\mathrm{H}\left(\mathrm{t}+\Delta \mathrm{t}-\mathrm{n} / \mathrm{f}_{\mathrm{c}}\right)\right] \times\left(1-\cos 2 \pi \mathrm{f}_{\mathrm{c}}(\mathrm{t}+\Delta \mathrm{t}) / \mathrm{n}\right) \times \sin 2 \pi \mathrm{f}_{\mathrm{c}}(\mathrm{t}+\Delta \mathrm{t})
$$

where $\mathrm{u}(\mathrm{t})$ is shown in Formula (6), $\alpha$ represents the attenuation coefficient, $0<\alpha<1, \Delta \mathrm{t}$ is the time delay, and $\Delta t>0$. The monitoring signal model is the sum of Formulas (6) and (7) and can be expressed as

$$
\mathrm{U}(\mathrm{t})=\mathrm{u}(\mathrm{t})+\mathrm{u}^{\prime}(\mathrm{t})=\mathrm{u}(\mathrm{t})+\alpha \mathrm{u}(\mathrm{t}+\Delta \mathrm{t})
$$

In this way, through the change of $\alpha$ and $\Delta t$ parameters in Formula (8), the influence of damage location and scattering wave energy on the instantaneous phase of monitoring signal can be analyzed.

\subsection{Experiment of the Instantaneous Phase of Monitoring Signal Model}

The monitoring model was used to analyze the instantaneous phase change by the damage scattering wave. The experiment required the assignment of $\alpha$ and $\Delta t$. According to the above description of the monitoring signal model, $\alpha$ is defined as the ratio of the amplitude of the scattering wave to the direct wave. Generally, the energy of scattering wave is far less than that of the direct wave. According to the two experiments in this paper, the ratio of the maximum amplitude of scattering wave(A1) to that of the direct wave(A0) was 0.06 and 0.1 (as shown in the figures in Section 3.3). Therefore, the range of the $\alpha$ value was set to $0.01-0.1$, and the change step was 0.01 . $\Delta$ t should be given different values to simulate the scattering wave coming from different damage locations. Its range is equal to the time range of the direct wave (0-1 s), and the change step was $0.01 \mathrm{~s}$. There is no necessary correlation between $\Delta t$ and $\alpha$. Thus, their correlation is weak and can be ignored. Therefore, when 
analyzing the influence of one variable on the instantaneous phase, another variable is set as a constant. The advantage of this approach is that the influence of variables $(\alpha$ or $\Delta t)$ on the instantaneous phase change can be demonstrated. In the following experiments, when analyzing the influence of the superposition site on instantaneous phase change, $\alpha$ was set to a constant, which was between 0.01 and 0.1 , and the step of $\Delta \mathrm{t}$ increased gradually by $0.01 \mathrm{~s}$. When analyzing the influence of attenuation on instantaneous phase change, the typical superposition site was chosen as the research object, and the step of $\alpha$ increased gradually by 0.01 .

The experiment is shown in Figure 3. Equations (6) and (7) were used to generate a direct wave and a scattering wave. The attenuation coefficient was set to 0.05 , and the simulated scattering wave is shown in Figure 3aI. The maximum point of the scattering wave (indicated by the triangle (" $\Delta$ ") in Figure 3aI) was taken as the observation point of scattering signal moving right, and the superposition site was defined as the intersection point between the projection of the maximum point $($ " $\Delta$ ") on the abscissa and the direct wave (indicated by the asterisk ("**) in Figure 3aI). The initial time coordinate of the observation point was $\mathrm{t}_{0}$, and the $\Delta \mathrm{t}$ in the monitoring signal model was the time difference between the superposition site and $t_{0}$. The instantaneous phase of the direct wave is shown in Figure 3aII. In Figure $3 b-d$, the instantaneous phase of change of the monitoring signal after scattering wave superposition is shown. The asterisks in subfigure I represent the superposition site; in subfigure II, the instantaneous phase of the signal after the superposition with the scattering wave is demonstrated; and subfigure III denotes a locally enlarged view of subfigure II. In Figure $3 \mathrm{~b}-\mathrm{d}$, the arrow in subfigure I indicates the moving direction of the superposition site (the moving interval is $0.01 \mathrm{~s}$ ), while the arrow in subfigure III indicates the corresponding change in the direction of the instantaneous phase. The effect of the attenuation coefficient $\alpha$ is shown in Figure $3 \mathrm{~b}-\mathrm{d}$. The red triangles in Figure 3eI-fI are the two superposition sites. When $\alpha$ increased from 0.01 to 0.1 (the step size was 0.01), the 10 scattering waves are shown in Figure 3(eI,fI). The change of the instantaneous phase with the attenuation coefficient increasing is shown in Figure 3(eII,fII). The subfigures III denote a locally enlarged view of subfigure II, and the arrow indicates the change direction on the instantaneous phase when the attenuation coefficients increased.

The experimental scheme constructed in this paper shows the effect of the scattering signal on the instantaneous phase of the signal. As shown in Figure $3 b-d$, the effect is manifested in two aspects: one is whether the instantaneous phase of the superimposed scattering signal is larger or smaller than the original instantaneous phase; the other is the degree of the instantaneous phase change after the superimposed scattering signal. As shown in Figure 3b, when the superposition site was between the maximum and the minimum, the instantaneous phase of the signal was smaller than the original instantaneous phase. As shown in Figure 3c, when the superposition site was between the minimum and the maximum, the instantaneous phase of the signal was larger than the original instantaneous phase. When the superposition site was again between the maximum and minimum, as shown in Figure 3d, the instantaneous phase was again smaller than the original instantaneous phase. When the superposition site was again between the minimum and the maximum, the instantaneous phase was again larger than the original instantaneous phase. From the description above, it can be concluded that whether the instantaneous phase becomes larger or smaller is absolutely determined by the extreme interval of the superposition location. The instantaneous phase distortion trend can be obtained from Figure $3 b-d$. When the scattering wave and the direct wave tended to be antiphase, the instantaneous phase deformation increased gradually; on the contrary, the deformation tended to slow down. The above experimental phenomenon would be repeated if the scattering wave continues to shift to the right. In Figure $3 \mathrm{e}, \mathrm{f}$, the effect of scattering wave attenuation on the instantaneous phase is illustrated. The experimental results show that the attenuation degree could not change the size relationship between the instantaneous phase after superimposing the scattering wave and the original instantaneous phase, but it mainly affected the time range of instantaneous phase deformation. When $\alpha$ was large, the range of the instantaneous phase deformation spreading along the time axis was also larger; on the contrary, the range along the time axis was smaller. Similar results could be 
obtained at the other superposition site. The following conclusions can be drawn from the analysis of the instantaneous phase change shown in Figure 3. When the superposition location was between the maximum and the minimum, the instantaneous phase of the monitoring signal model became larger than that of the direct wave. When the superposition location was between the minimum and the maximum, the instantaneous phase of the monitoring signal model became smaller than that of the direct wave. When the superposition site made the scattering wave and the direct wave tend to be antiphase, the instantaneous phase deformation of the monitoring signal model became larger. The attenuation degree of the scattering wave only affected the range of the instantaneous phase deformation on the time axis and did not change the size relationship.
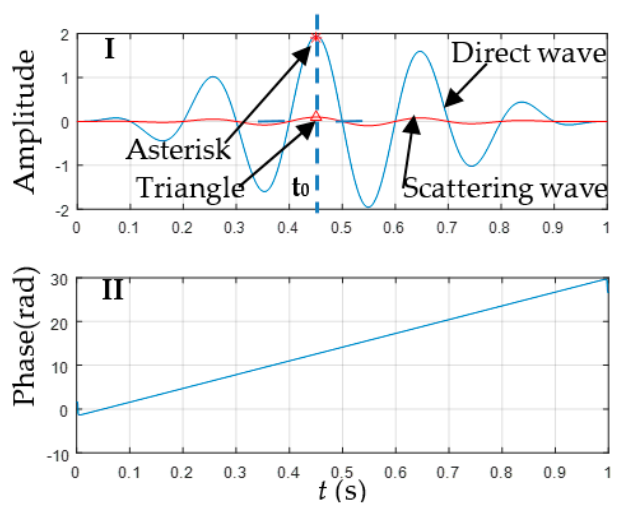

(a)

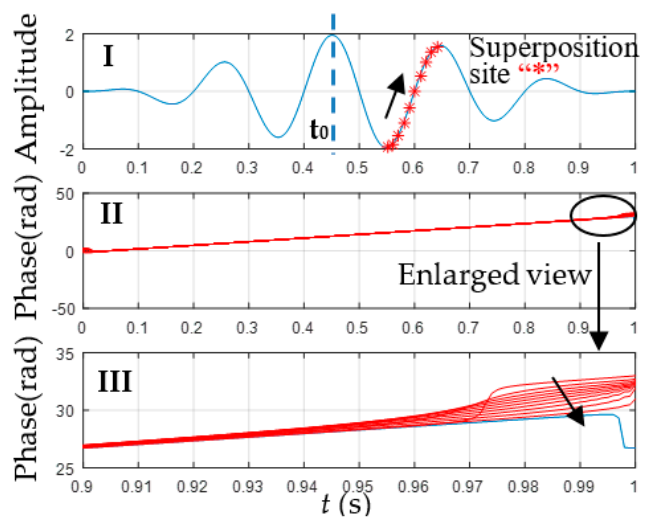

(c)

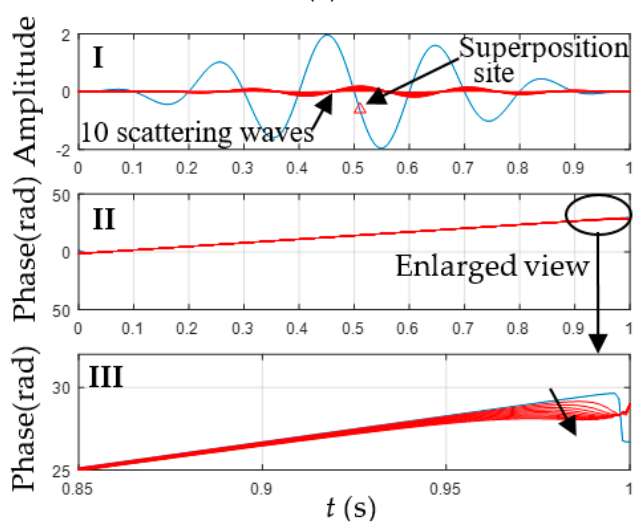

(e)

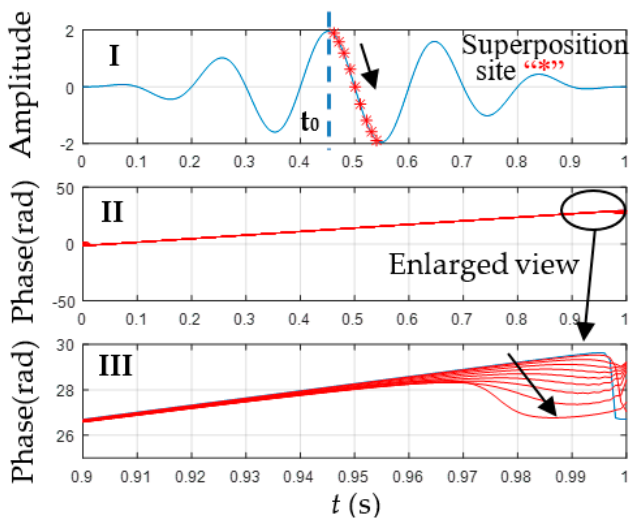

(b)

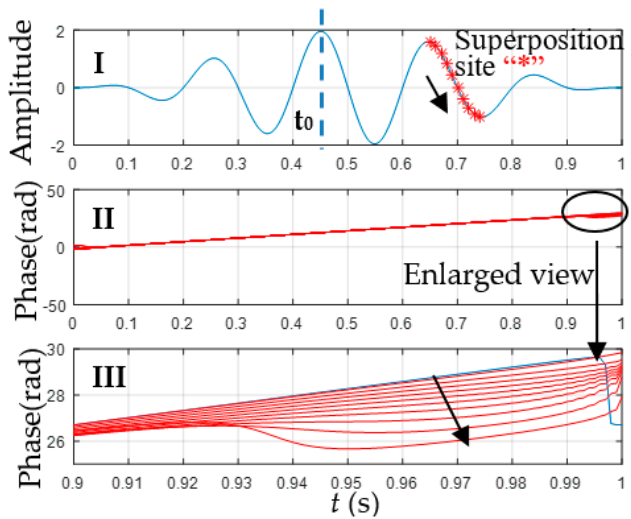

(d)
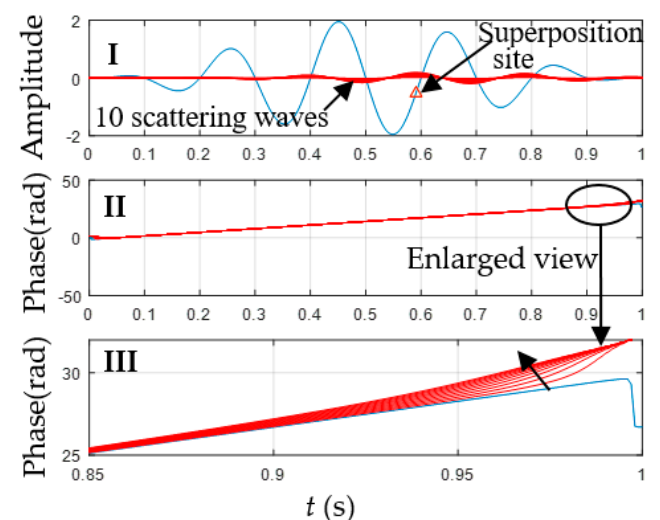

(f)

Figure 3. The experiment of the instantaneous phase change of the monitoring signal model. (a) Subfigure (I) shows the direct wave and the scattering wave, and subfigure (II) shows the instantaneous 
phase of the direct wave. (b) The superposition site occurred between the maximum and minimum. Subfigure (I) shows the superposition, and subfigures (II,III) show the instantaneous phase change and a locally enlarged view. (c) The superposition site occurred between the minimum and maximum. Explanations of the subfigures are the same as (b). (d) The superposition site returns between the maximum and minimum. Explanations of the subfigures are the same as (b). (e) The instantaneous phase change with the attenuation coefficient increase when the superposition site occurred between the minimum and maximum. Subfigure (I) shows 10 scattering signals with different attenuation coefficients and superposition sites, and subfigures (II,III) show the instantaneous phase change and a locally enlarged view. (f) The instantaneous phase changes with the attenuation coefficient increase when the superposition site occurred between the minimum and maximum. Explanations of the subfigures are the same as (e).

The following conclusions can be drawn from the analysis of the monitoring signal model. Instantaneous phase change means that the monitoring signal overlaps the scattering signal, and the superposition site of the scattering signal is dominant to the change direction and the local deformation of the instantaneous phase. The energy of the scattering wave only affected the range of deformation in time. This paper studies the instantaneous phase of the signal model and reveals the causal relationship between the instantaneous phase change and the superposition of scattering waves. Based on this relationship, it is feasible to diagnose the structure health of the plate according to the instantaneous parameter characteristics of the signal.

\subsection{Analysis of the Instantaneous Phase of the Monitoring Signal}

An experimental setup was built for the active Lamb wave based monitoring, and an epoxy glass fiber plate was used as the object of monitoring. The damage types of the composite plate include matrix fracture, fiber breakage, and internal delamination [30]. The damage monitoring method proposed in this paper focuses on damage with an explicit damage boundary that can easily scatter Lamb waves, such as a matrix fracture, fiber breakage, and crack. In the following experiment, a through-hole was used to simulate the scattered waves of these damages. Both the actuator and the sensor were PZTs with a diameter of $8 \mathrm{~mm}$ and a thickness of $0.5 \mathrm{~mm}$. The material of PZT was lead zirconate titanate. The epoxy glass fiber plate used in the experiment was $2 \mathrm{~mm}$ thick and the center frequency of excitation signal was $100 \mathrm{kHz}$ and $150 \mathrm{kHz}$. Thus, there were two fundamental modes of Lamb wave propagated in the plate. If the distance between the actor and the sensor was short, then the waves of these two modes would mix, and if the distance was long, then the attenuation of the waves would be large. According to the experimental conditions, the propagating distance of the wave was suggested to be set at $15-60 \mathrm{~cm}$ to ensure that the sensing signal was single-mode and had enough energy.

The experimental setup is presented in Figure 4a. The center distance between the actuator (PZT1) and sensor (PZT2) was $40 \mathrm{~cm}$. The damage in the plate was a through-hole with a diameter of about $6 \mathrm{~mm}$, which was approximately at a distance of $20 \mathrm{~cm}$ and $25 \mathrm{~cm}$ from PZT1 and PZT2, respectively. Equation (6) was used as the mathematical expression of the excitation signal (with a center frequency of $100 \mathrm{kHz}$ and $150 \mathrm{kHz}$ and five peaks). The actuator PZT1 was excited once, and the monitoring system collected the transmitted Lamb wave by sensor PZT2, performing the one-time signal data collection. To reduce the impact of random noise on results, each monitoring signal denoted the average value of 20 single successive received signals. To ensure the reliability of the analysis results, 10 monitoring signals were respectively collected under the condition of both plate health and damage. Using 10 signals was a strategy for experimental data analysis. The purpose was to observe whether the instantaneous parameter changes of the signal caused by the damage were ubiquitous in the signal and whether the difference of instantaneous parameters between the same state signals could conceal the instantaneous parameter changes of the signal caused by damage. The response of the plate structure under excitation by the signal with the center frequency of respectively $100 \mathrm{kHz}$ and $150 \mathrm{kHz}$ is presented in Figure $4 b$, wherein the solid line denotes the response when there was not any damage, 
and the dashed line denotes the response when there was damage. Following the analysis in Section 2.2, only the Lamb waves labeled as Wave packet 1 in Figure $4 \mathrm{~b}$ were used to study the instantaneous phase and the marginal spectrum.

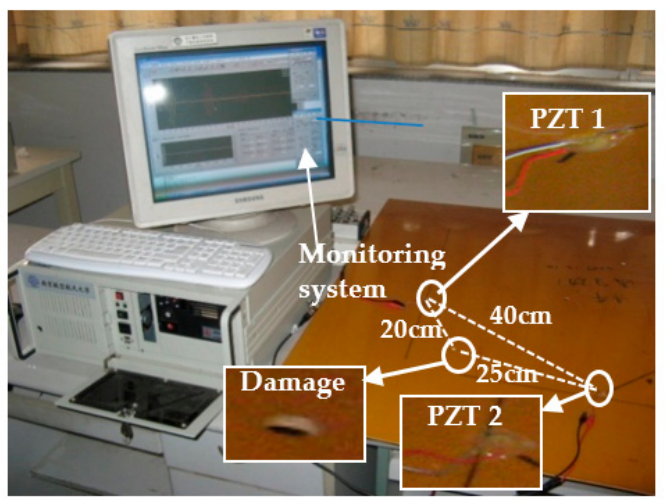

(a)

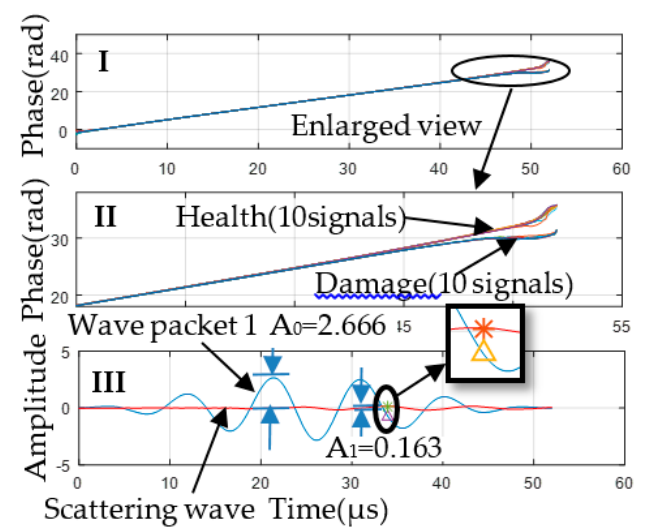

(c)
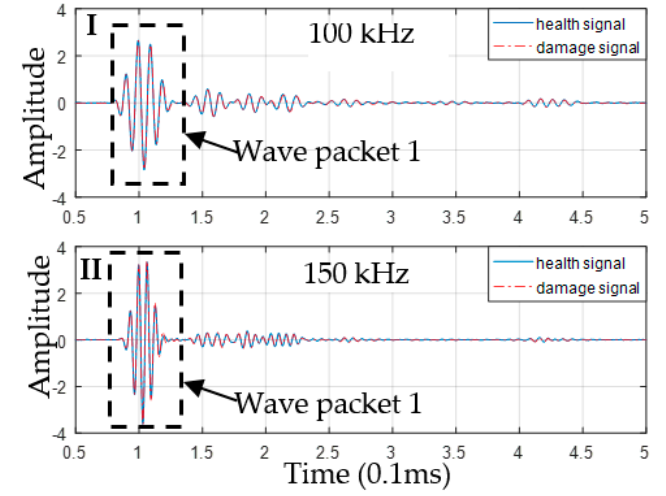

(b)

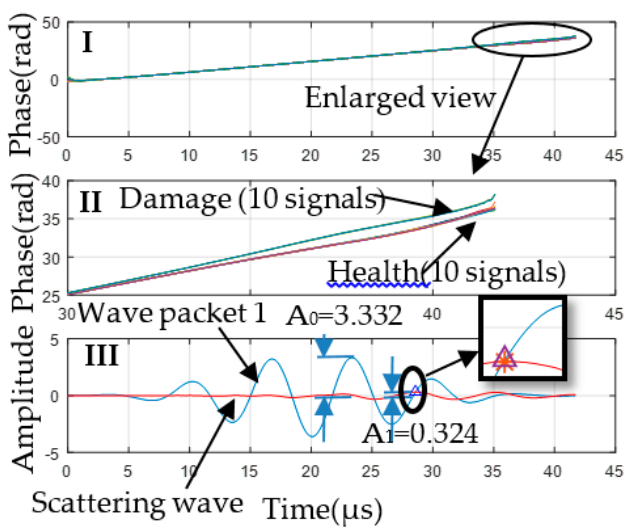

(d)

Figure 4. The sensing signal and the instantaneous phase in the active Lamb wave-based monitoring of the epoxy glass fiber plate structure. (a) Experimental setup for the active Lamb wave-based monitoring of the epoxy glass fiber plate. (b) Sensing signal of the active Lamb wave-based monitoring, and subfigures (I,II) show the response of the plate structure excited by the signal with the center frequency of $100 \mathrm{kHz}$ and $150 \mathrm{kHz}$, respectively; (c) The monitoring signal with a center frequency of $100 \mathrm{kHz}$ and the instantaneous phase, and subfigure (I) shows the instantaneous phase change, subfigure (II) denotes a locally enlarged view of (II), and subfigure (III) shows some information for the monitoring signal. (d) The monitoring signal with a center frequency of $150 \mathrm{kHz}$ and the instantaneous phase. The explanation of the subfigures (I-III) is the same as (c).

The instantaneous phase analysis is shown in Figure $4 \mathrm{c}, \mathrm{d}$ with a center frequency of $100 \mathrm{kHz}$ and $150 \mathrm{kHz}$, respectively. In Figure 4c,d, subfigure I presents the instantaneous phase of 10 monitoring signals after damage and 10 monitoring signals before damage; subfigure II denotes the locally enlarged view of subfigure I; subfigure III shows the information for the monitoring wave. As shown in Figure $4 \mathrm{c}$,dII, the instantaneous phase of the 10 damage signals presented the same change concerning the instantaneous phase of 10 health signals, which indicates that the change was caused by a specific cause rather than a random signal. This change indicates that the monitoring signal was mixed with the scattering wave, and then it can be inferred that there was damage in the propagation path of the Lamb wave in a certain direction, which means that the plate structure was damaged. The damage diagnosis result obtained from the instantaneous phase change was consistent with the actual situation of the plate. According to Figure 4c,dIII, the direction and deformation of the instantaneous phase after damage could be obtained. In Figure 4cII, the instantaneous phase after the damage was smaller 
than the original instantaneous phase. According to the relationship between the superposition site and the direction of the instantaneous phase change obtained in Section 3.2, the superposition site of the scattering wave should be between the maximum and minimum of the direct wave. The real scattering wave and direct wave were used to verify this inference. In Figure 4cIII, the Wave packet of the direct wave is labeled "Wave packet 1", and the damage scattering wave is labeled as "Scattering wave." According to the definition of the superposition site in Section 3.2, the maximum point of the "Scattering wave" is marked as "*", and the intersection point between the projection of this maximum "*" point on the abscissa, and the direct wave is the superposition site, which is marked as " $\Delta$ ". The local magnification in Figure $4 \mathrm{cIII}$ shows that " $\Delta$ " is indeed between the maximum and minimum of the direct wave. In Figure 4dIII, based on the same processing and analysis, the instantaneous phase after the damage is larger than the original phase, and the superposition position of scattering wave should be between the minimum and maximum. The local magnification in Figure 4dIII shows that " $\Delta$ " is indeed between the minimum and maximum of the direct wave. The instantaneous phase analysis results of the two experiments are consistent with the relation between the instantaneous phase change direction and the scattering wave superposition summarized in Section 3.2. The instantaneous phases are shown in Figure 4(cII,dII), and their deformation degree is different from the original instantaneous phase shape. From the analysis of the instantaneous phase change in Section 3.2, when the superposition site makes the phase difference between the scattering wave and the direct wave tend to be in antiphase, the greater the deformation degree is, and vice versa. Compared with the superposition site in Figure 4dIII, the superposition site in Figure 4cIII made the scattering wave closer to the antiphase of the direct wave, and thus the local deformation of its instantaneous phase should be larger. Large deformation is beneficial for improving the recognition of instantaneous phase change. Moreover, the influence of scattering wave energy on the local deformation range of the instantaneous phase was discussed in Section 3.2. Although the scattering wave energy ratio $(\mathrm{A} 1 / \mathrm{A} 0=0.1)$ in Figure $4 \mathrm{dIII}$ is higher than that in Figure 4cIII $(\mathrm{A} 1 / \mathrm{A} 0=0.06)$, there is no significant difference between their instantaneous phase deformation ranges. It is considered that the damage scattering wave is the rescattering of the wave, and its energy is already very small; thus, its effect on the instantaneous phase change is limited. That is to say, in the case of the same or close energy, the effect of phase difference on the instantaneous phase change is higher than that of energy. This is also consistent with the conclusion that the superposition site of the scattering wave dominates the instantaneous phase change in Section 3.2.

The above analysis based on the instantaneous phase diagnosis of plate structure damage shows that both the real damage monitoring experiment and the signal model analysis in Section 3.2 support this paper's proposal that the plate structure health monitoring can be realized through the change of the instantaneous phase of a single sensing signal. Furthermore, the research content shows that the phase difference has a direct impact on the instantaneous phase deformation. Its role in the damage diagnosis method based on the instantaneous phase change is equivalent to the role of the difference signal energy in the damage diagnosis method based on the difference of signal. The appropriate phase difference can improve the identification of the instantaneous phase change. Two experiments showed that the active Lamb wave based plate structure health monitoring can utilize multiple center frequency excitation to make the phase difference between the damage scattering wave and the direct wave as close as possible to the antiphase, thus improving the identification of instantaneous phase change.

\section{Analysis of Marginal Spectrum Change of an Active Lamb Wave}

\subsection{Preliminary Analysis of the Marginal Spectrum}

The instantaneous frequency is defined by HHT theory as a derivative of the instantaneous phase [20]. An analysis of the experimental results shows that the superposition of the scattering signal could cause instantaneous phase change, and thus the instantaneous frequency would also change. The experimental results of the signal with a center frequency of $150 \mathrm{kHz}$, which is shown in Figure 4, 
are presented in Figure 5. Figure 5aI shows the time sequence of the Lamb wave packet before and after the damage (10 signals for each condition). Figure 5aII displays the instantaneous frequency obtained from the signal that is presented in Figure 5aI. Figure 5aII shows that there was an obvious frequency change in the interval from $2.5 \mu$ s to $3.5 \mu \mathrm{s}$. Following Equation (5), the marginal spectrum was defined as the integral of the IMF's Hilbert spectrum over time, describing the relationship between the signal energy and its instantaneous frequency. When the instantaneous frequency changed, the energy distribution also changed. Therefore, the damage scattering waves could change the distribution of the Lamb wave marginal spectrum in the frequency domain. Figure 5bI shows the IMFs obtained by decomposing the signal presented in Figure 5aI by the EMD. Figure 5bI shows that the energy was mainly concentrated in the first few order IMFs. Considering that the EMD decomposition was performed from high frequencies to low frequencies, the other order IMFs did not represent effective frequency components, and their energy was relatively small. In this work, only the first three order IMFs were used to determine the marginal spectrum. Figure 5bII shows the obtained marginal spectrum, presenting the change before and after damage.

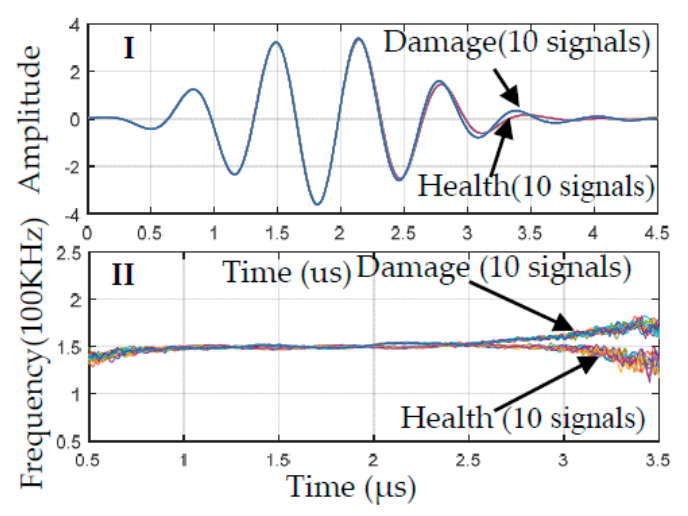

(a)

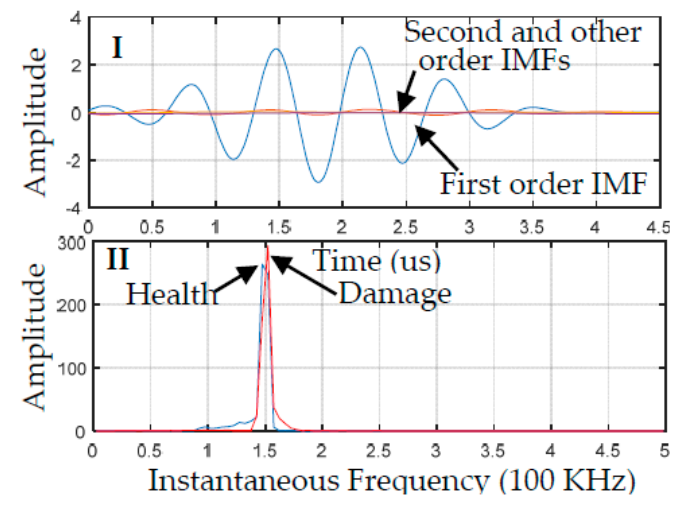

(b)

Figure 5. Instantaneous frequency and marginal spectrum of the Lamb wave packet with a center frequency of $150 \mathrm{kHz}$. (a) Sequence diagram and instantaneous frequency of the wave packet, and subfigure (I) shows the wave packet before and after damage; subfigure (II) shows the instantaneous frequency before and after damage. (b) The intrinsic mode function (IMF) and marginal spectrum of the wave packet. Subfigure (I) shows the IMFs after empirical mode decomposition (EMD) decomposition of the wave packet, and subfigure (II) shows the marginal spectrum of the wave packet before and after damage.

The explanation given above is only a preliminary illustration that the damage scattering signal can change the distribution of the Lamb wave marginal spectrum in the frequency domain. A detailed explanation of the causes is given below.

\subsection{Application Analysis of Marginal Spectrum Change in Plate Structural Health Monitoring}

In the calculation of the marginal spectrum of the signal, the first step is to decompose the signal into multiple order IMFs by using the EMD. The EMD is an adaptive signal decomposition method based on the extreme signal points [20]. It is impossible to forecast the influence of minor changes in the extreme points on the IMFs, because the EMD process is complicated and includes interpolation, end-point processing, and IMF judgment. Namely, the signals are highly consistent from the sequence perspective, but they have randomly scattered extreme points, which may result in poor consistency in the IMFs obtained through the decomposition. The inconsistency degree can be so high that it can be impossible to analyze the marginal spectrum change of a signal before and after damage. Therefore, to guarantee the consistency in the IMFs acquired through the EMD process, the randomly scattered extreme points must be filtered out. Notably, in this study, the phase shift information of 
the instantaneous frequency of the signals that cannot be generated by a filter is analyzed, and thus the zero-phase filtering method must be used [31,32]. Besides, Wave packet 1 of the health sensing signal with a center frequency of $100 \mathrm{kHz}$, shown in Figure 4bI, is used as an example. The first-order IMFs obtained by the EMD before filtering out randomly scattered extreme points are presented in Figure $6 a$, wherein it can be seen that there was a poor consistency between the first-order IMFs of the ten same-condition signals. The first-order IMFs obtained by the EMD after filtering out randomly scattered extreme points are presented in Figure $6 \mathrm{~b}$, wherein it can be seen that the consistency between the IMFs of the ten same-condition signals could be achieved.

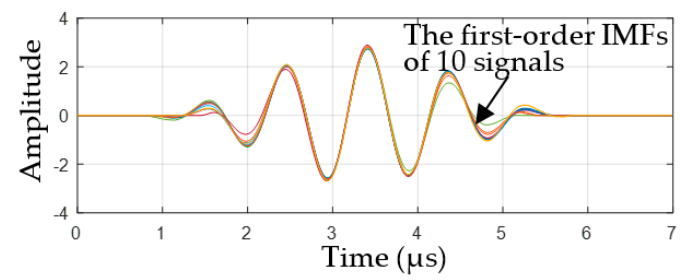

(a)

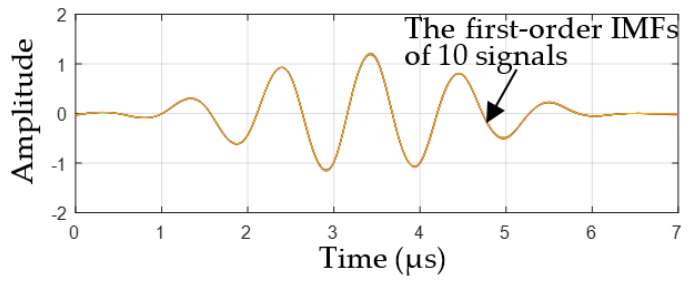

(b)

Figure 6. The first-order IMFs of two decompositions. (a) The first-order IMFs obtained by decomposition without zero-phase filtering; (b) The first-order IMFs obtained by decomposition with zero-phase filtering.

Under the premise of ensuring consistency in signal decomposition, the Lamb wave packet presented in Figure $4 b$ was decomposed into multiple IMFs. As shown in Figures $5 b$ and $6 b$, the signal energy was mainly concentrated in the first several order IMFs. According to the description in Section 4.1, only the first three orders of IMFs were needed to calculate the marginal spectrum, which can be further used to study the changes in the Lamb wave marginal spectrum before and after damage. Since the energy was mainly concentrated near the center frequency, the marginal spectrum corresponding to the frequency band near the center frequency was used. To compare the distribution of the marginal spectrum in the frequency domain before and after the damage more easily, the frequency band was divided into several continuous bands, and then the marginal spectrum amplitudes corresponding to all the frequencies in each frequency band were considered to be the marginal spectrum amplitudes of that band. To make the experimental data analysis conclusion reflect the marginal spectrum variation characteristics of the active Lamb wave signal after damage as closely as possible, the number of damage and health state signals used in the analysis was 10 . The 10 marginal spectral amplitudes before and after the damage of the same frequency band are displayed in the same subfigure.

The marginal spectrum of the Lamb wave with a center frequency of $100 \mathrm{kHz}$ is presented in Figure $7 \mathrm{a}$, where the abscissa corresponds to the signal number and the ordinate corresponds to the amplitude of the marginal spectrum. The marginal spectrum was concentrated in the frequency band $80-120 \mathrm{kHz}$, which was divided into five continuous bands as follows: $8088 \mathrm{kHz}, 88-96 \mathrm{kHz}$, 96-104 kHz, 104-112 kHz, and 112-120 kHz. The number of corresponding figures of each band was from I to V. The marginal spectrum amplitude in each frequency band is presented in Figure $7 \mathrm{a}$, where “*” represents 10 healthy signals and " $\Delta$ " represents 10 damage signals. For both health signals and damage signals, the marginal spectral amplitudes of the last two bands $(104-112 \mathrm{kHz}$ and $112-120 \mathrm{kHz}$, see subfigures VI-V in Figure 7a) were too small, and thus they were not involved in the following analysis. In Figure 7aI-III, the marginal spectrum amplitude (" $\Delta$ " in the figures) after the damage is different from that before the damage. In subfigures I and II, the corresponding frequency bands were $80-88 \mathrm{kHz}$ and $88-96 \mathrm{kHz}$, and the marginal spectrum amplitude after damage increased. In subfigure III, the corresponding frequency band was $96-104 \mathrm{kHz}$, and the marginal spectrum amplitude after damage decreased. Whether it increased or decreased, the change direction of the 10 marginal spectra in the same frequency band was the same after damage, and the change amount was also consistent, 
which indicates that the marginal spectral change was not caused by random signals. According to the actual state, in Figure 7a, the change in the marginal spectrum of the active Lamb wave monitoring signal could only be caused by the scattering signals from the plate structure damage.
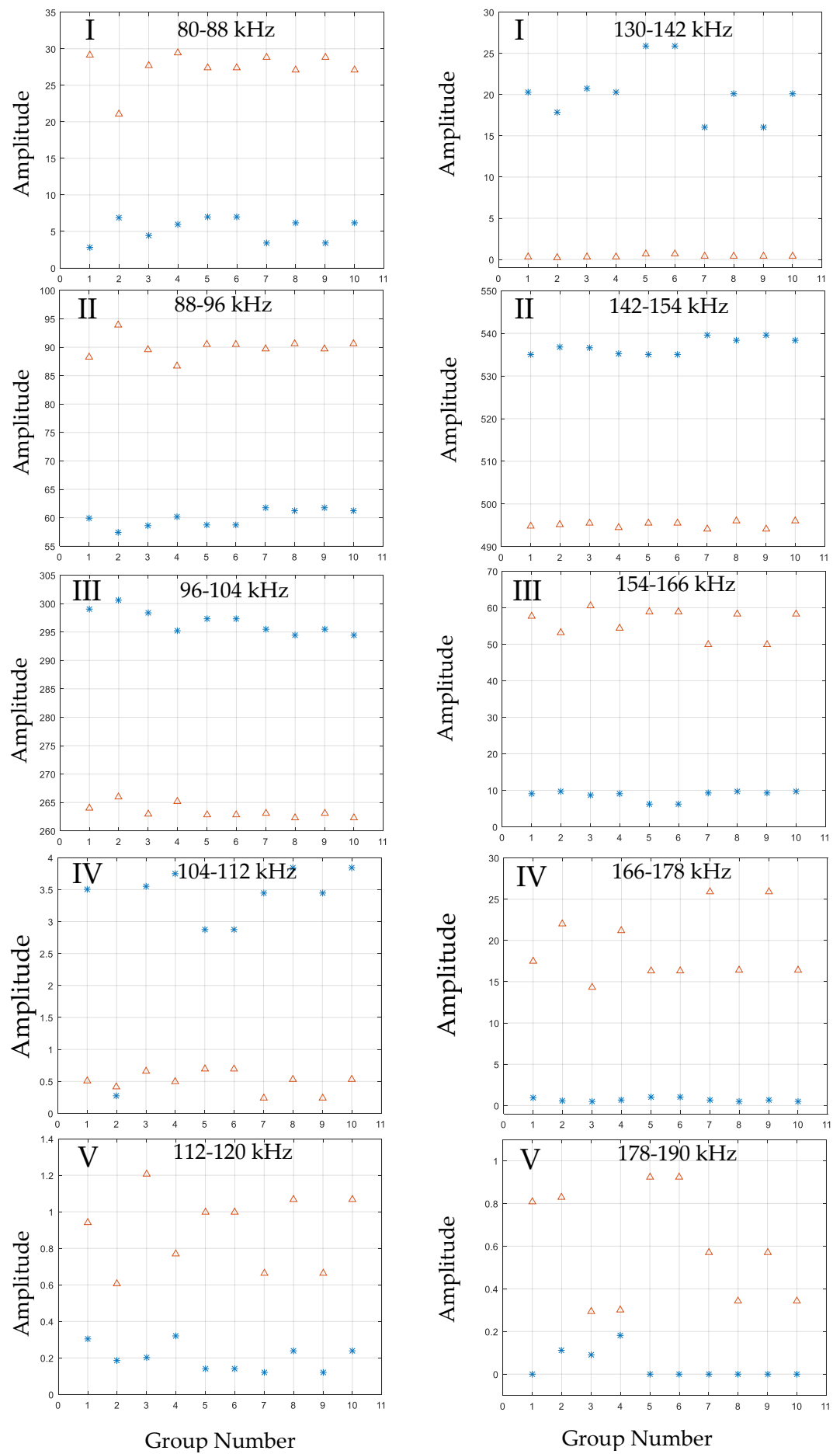

(a)

(b)

Figure 7. The frequency range around the two excitation frequencies is divided into five frequency bands and the marginal spectrum amplitude of the Lamb wave in each frequency band. "**" is the marginal spectrum amplitude before the plate damage, and " $\Delta$ " is the marginal spectrum amplitude after the plate damage. Subfigures (I-V) relate to the five frequency bands. (a) The marginal spectrum of $100 \mathrm{kHz}$ excited Lamb waves; (b) The marginal spectrum of $150 \mathrm{kHz}$ excited Lamb waves. 
The marginal spectrum of the Lamb wave with a center frequency of $150 \mathrm{kHz}$ is presented in Figure $7 b$. Like the analysis process in Figure 7a, the efficiency frequency band $130-190 \mathrm{kHz}$ was divided into five successive bands, labeled from I to V. Frequency band 178-190 kHz was not involved in the following analysis because the energy was too small. By comparing Figure 7a,b, their marginal spectral energy diffusion directions were different in addition to their different center frequencies. In subfigures I and II, the corresponding frequency bands were $130-142 \mathrm{kHz}$ and $142-154 \mathrm{kHz}$, and the marginal spectrum amplitude wave decreased after damage. In subfigures III and IV, the corresponding frequency bands were $154-166 \mathrm{kHz}$ and $166-178 \mathrm{kHz}$, and the marginal spectrum of Lamb wave increased after damage. The change characteristics of the marginal spectrum were like those of Figure 7a. In each subfigure I-IV, the change direction of the marginal spectrum was the same, and the variation amount of the marginal spectrum was consistent. Figure $7 \mathrm{~b}$ shows that the marginal spectrum changed significantly after damage, and the abnormal of active Lamb wave could be judged to diagnose the plate structure damage.

The above marginal spectrum analysis of two different center frequencies shows that the change of the active Lamb wave marginal spectrum after the damage had some characteristics. The marginal spectrum change was not limited to a certain frequency band but rather existed in several continuous frequency bands. In the effective frequency range, the marginal spectrum of the local frequency band increased or decreased after damage, but the whole frequency range did not increase or decrease. As shown in Figure 7, within each local frequency band, the amplitude of the 10 marginal spectra changes in the same direction had approximately the same amount, indicating that the marginal spectrum could reliably reflect the anomaly of the active Lamb wave. According to the above characteristics, it is suggested to use the comprehensive situation of the marginal spectrum amplitude in multiple continuous frequency bands, such as the amplitude change or amplitude ratio between frequency bands, to diagnose the structural damage of the plate, which can improve the diagnostic accuracy. These changes in the marginal spectrum of signals after damage can be explained by the instantaneous phase changes of signals, and the relationship between them is analyzed in Section 5.

\section{Analysis of the Relationship between the Instantaneous Phase and Marginal Spectrum}

In Figure 7, by comparing the change of marginal spectrum amplitude before and after damage, it can be seen that the marginal spectrum of the Lamb wave with a center frequency of $100 \mathrm{kHz}$ diffused to low frequencies after the damage of the plate structure while that of the Lamb wave with a center frequency of $150 \mathrm{kHz}$ diffused to high frequencies. Such results could not be explained only by the change in the marginal spectrum. However, by considering the change in the instantaneous phase, these two different directions of diffusion could be explained.

As presented in Figure 4c, when the center frequency of the monitoring signal was $100 \mathrm{kHz}$, the damage scattering wave reduced the instantaneous phase of the Lamb wave, that is, the slope of the relationship curve between the time and instantaneous phase decreased. According to Equation (3), the slope denoted the instantaneous frequency. Therefore, the active Lamb wave monitoring signal with a center frequency of $100 \mathrm{kHz}$, after superimposing the damage scattering signal, decreased and the corresponding frequency to the marginal spectrum also decreased. This explains why the marginal spectrum of the Lamb wave diffused to low frequencies after the plate structure damage, as shown in Figure 7a. When the center frequency of the monitoring signal was $150 \mathrm{kHz}$, the damage scattering wave increased the instantaneous phase of the Lamb wave. As shown in Figure 4c, the instantaneous frequency of the monitoring signal increased. Therefore, the frequency corresponding to the marginal spectrum also increased, which explains why the marginal spectrum of the Lamb wave diffused to high frequencies after plate structure damage, as shown in Figure $7 b$. In Figure $4 c, d$, the instantaneous phase change is smooth, and thus the instantaneous frequency change is continuous. It has been shown that instantaneous frequency change caused the marginal spectrum to diffuse towards low or high frequencies. The marginal spectrum diffusion after the damage was in the range around the center frequency only because the instantaneous frequency change was continuous, which shows 
that the marginal spectrum changed in multiple continuous local frequency bands, and the marginal spectrum amplitude increased and decreased in some local frequency bands.

The above analysis of the monitoring signal shows that, in the active Lamb wave based monitoring of the plate structural health, the change in the signal instantaneous phase could indicate the change in the signal marginal spectrum and vice versa. The damage scattering wave caused the change of the instantaneous phase of the monitoring signal, and the change of the instantaneous phase also showed the existence of the damage scattering wave. The monitoring method based on the difference between the monitoring signals before and after damage illustrated the change of the signals after damage from the time domain. In this paper, the monitoring methods based on instantaneous phase change and marginal spectrum change of monitoring signal described the change of the signal after damage from the frequency domain and energy. These three monitoring methods are all based on the direct wave changes caused by damage scattered signals. They are different explanations of the same physical phenomenon. The research content and analysis results also show that they are interrelated. Therefore, it is reliable and feasible to use the instantaneous parameter change of active Lamb wave to realize plate structure health monitoring.

\section{Conclusions}

The effect of damage scattering signals on the instantaneous parameter characteristics of active Lamb waves was studied in this paper, which proved the feasibility of extracting damage information from the instantaneous phase and marginal spectral changes of a single sensor signal. This paper provides a diagnostic method for active Lamb wave based monitoring based on instantaneous parameter characteristic changes. In the active Lamb wave based monitoring of plate structure health, the excitation wave and the propagation distance of the response wave are constant, and thus the instantaneous feature of sensing signal is also fixed. This is the basis of the monitoring method proposed in this paper. To prove the effectiveness of the proposed monitoring method, first, a damage monitoring signal model was established to study the relationship between the superposition position of the damage scattering signals and the instantaneous phase change of the monitoring signals. Then, the reasons for the instability of EMD decomposition of signals in the same state were studied and methods to improve the decomposition stability were proposed. Based on these studies, the relationship between damage and the change of the instantaneous phase and marginal spectral was revealed. This paper further analyzes the relationship between the marginal spectrum change and the instantaneous phase, which indicates that the marginal spectrum change of an active Lamb wave is not disordered but rather related to instantaneous phase change. The research results show that there is a causal relationship between the instantaneous parameter characteristics of active Lamb wave and the damage scattering signal. It is feasible to realize the health monitoring of plate structure based on the change of instantaneous parameter characteristics of an active Lamb wave.

This damage monitoring method is suitable for the damage that can scatter the propagation wave, such as matrix damage and fiber fractures of composite material and crack and hole damage of metal plates whether the damage is visible or not. This diagnosis method can describe the damage information change in monitoring signals from two aspects: instantaneous phase and marginal spectral distribution. Compared with the previous diagnosis method that only depends on the difference between monitoring signals, this diagnosis method can reduce the misdiagnosis rate, does not need to be strictly consistent with each acquisition trigger threshold, improves the anti-interference ability, and reduces the hardware requirements of the monitoring system.

Author Contributions: Conceptualization, B.X.; methodology, B.X. and Y.S.; software, P.L.; validation, Q.C. and Y.S.; formal analysis, B.X.; writing—original draft preparation, B.X.; writing—review and editing, P.L.; supervision, M.W. All authors have read and agreed to the published version of the manuscript.

Funding: This research was funded by Major Project of Natural Science Research of Jiangsu Province Universities (19KJA410001) and Scientific Research Foundation of Nanjing Institute of Technology (CKJB201808).

Conflicts of Interest: The authors declare no conflict of interest. 


\section{References}

1. Senyurek, V.Y. Detection of cuts and impact damage at the aircraft wing slat by using Lamb wave method. Measurement 2015, 67, 10-23. [CrossRef]

2. Li, F.; Peng, H.; Meng, G. Quantitative damage image construction in plate structures using a circular PZT array and lamb waves. Sens. Actuators A Phys. 2014, 214, 66-73. [CrossRef]

3. Haider, M.F.; Giurgiutiu, V. A Helmholtz Potential Approach to the Analysis of Guided Wave Generation during Acoustic Emission Events. J. Nondestruct. Eval. Diagn. Progn. Eng. Syst. 2017, 1, 021002. [CrossRef]

4. Xu, B.; Wu, J.; Wang, M. Mulan Study of modal acoustic emission to monitor the impact damage in a composite plate. J. Vibroeng. 2017, 19, 3335-3348. [CrossRef]

5. Baochun, X.; Shenfang, Y.; Mulan, W.; Lei, Q. Determining impact induced damage by lamb wave mode extracted by EMD method. Measurement 2015, 65, 120-128. [CrossRef]

6. Zelenyak, A.-M.; Hamstad, M.A.; Sause, M.G.R. Modeling of Acoustic Emission Signal Propagation in Waveguides. Sensors 2015, 15, 11805-11822. [CrossRef] [PubMed]

7. Haider, M.F.; Giurgiutiu, V. Analysis of axis symmetric circular crested elastic wave generated during crack propagation in a plate: A Helmholtz potential technique. Int. J. Solids Struct. 2018, 134, 130-150. [CrossRef]

8. Lin, X.; Yuan, F.G. Diagnostic Lamb waves in an integrated piezoelectric sensor/actuator plate: analytical and experimental studies. Smart Mater. Struct. 2001, 10, 907-913. [CrossRef]

9. Han, J.; Kim, Y. Time-frequency beam forming for nondestructive evaluations of plate using ultrasonic Lamb wave. Mech. Syst. Signal Process. 2015, 54-55, 336-356. [CrossRef]

10. Rathod, V.; Mahapatra, D.R. Ultrasonic Lamb wave based monitoring of corrosion type of damage in plate using a circular array of piezoelectric transducers. NDT E Int. 2011, 44, 628-636. [CrossRef]

11. An, Y.-K.; Kim, J.H.; Yim, H.J. Lamb Wave Line Sensing for Crack Detection in a Welded Stiffener. Sensors 2014, 14, 12871-12884. [CrossRef] [PubMed]

12. Zeng, L.; Lin, J.; Huang, L. A Modified Lamb Wave Time-Reversal Method for Health Monitoring of Composite Structures. Sensors 2017, 17, 955. [CrossRef] [PubMed]

13. Chen, J.; Yuan, S.; Qiu, L.; Cai, J.; Yang, W. Research on a Lamb Wave and Particle Filter-Based On-Line Crack Propagation Prognosis Method. Sensors 2016, 16, 320. [CrossRef] [PubMed]

14. Ihn, J.-B.; Chang, F.-K. Pitch-catch Active Sensing Methods in Structural Health Monitoring for Aircraft Structures. Struct. Health Monit. 2008, 7, 5-19. [CrossRef]

15. Huang, L.; Zeng, L.; Lin, J.; Luo, Z. An improved time reversal method for diagnostics of composite plates using Lamb waves. Compos. Struct. 2018, 190, 10-19. [CrossRef]

16. Moll, J.; Schulte, R.T.; Hartmann, B.; Fritzen, C.-P.; Nelles, O. Multi-site damage localization in anisotropic plate-like structures using an active guided wave structural health monitoring system. Smart Mater. Struct. 2010, 19, 126. [CrossRef]

17. Jia, H.; Zhang, Z.; Liu, H.; Dai, F.; Liu, Y.; Leng, J. An approach based on expectation-maximization algorithm for parameter estimation of Lamb wave signals. Mech. Syst. Signal Process. 2019, 120, 341-355. [CrossRef]

18. Law, L.-S.; Kim, J.H.; Liew, W.Y.; Lee, S.-K. An approach based on wavelet packet decomposition and Hilbert-Huang transform (WPD-HHT) for spindle bearings condition monitoring. Mech. Syst. Signal Process. 2012, 33, 197-211. [CrossRef]

19. Nalband, S.; Valliappan, C.; Prince, A.A.; Agrawal, A. Time-frequency based feature extraction for the analysis of vibroarthographic signals. Comput. Electr. Eng. 2018, 69, 720-731. [CrossRef]

20. Huang, N.E.; Shen, Z.; Long, S.R.; Wu, M.C.; Shih, H.H.; Zheng, Q.; Yen, N.-C.; Tung, C.C.; Liu, H.H. The empirical mode decomposition and the Hilbert spectrum for nonlinear and non-stationary time series analysis. Proc. R. Soc. A 1998, 454, 903-995. [CrossRef]

21. Liu, M.; Yang, J.; Cao, Y.; Fu, W.; Cao, Y. A new method for arrival time determination of impact signal based on HHT and AIC. Mech. Syst. Signal Process. 2017, 86, 177-187. [CrossRef]

22. Chen, H.; Yan, Y.J.; Jiang, J. Vibration-based damage detection in composite wingbox structures by HHT. Mech. Syst. Signal Process. 2007, 21,307-321. [CrossRef]

23. Biswas, A. Scale-location specific soil spatial variability: A comparison of continuous wavelet transform and Hilbert-Huang transform. Catena 2018, 160, 24-31. [CrossRef]

24. Susanto, A.; Liu, C.-H.; Yamada, K.; Hwang, Y.-R.; Sekiya, K. Application of Hilbert-Huang transform for vibration signal analysis in end-milling. Precis. Eng. 2018, 53, 263-277. [CrossRef] 
25. Chun, X.B.; Lan, W.M.; Qian, J. Application of Marginal Spectrum in Active Lamb Wave Damage Monitoring of Plate Struture. Appl. Mech. Mater. 2013, 490-491, 1698-1701.

26. Park, G.; Farrar, C.; Di Scalea, F.L.; Coccia, S. Performance assessment and validation of piezoelectric active-sensors in structural health monitoring. Smart Mater. Struct. 2006, 15, 1673-1683. [CrossRef]

27. Haider, M.F.; Poddar, B.; Giurgiutiu, V. Experimental validation of an analytical method to predict lamb wave scattering from a discontinuity. Smart Mater. Struct. 2018, 28, 015012. [CrossRef]

28. Haider, M.F.; Bhuiyan, M.Y.; Poddar, B.; Lin, B.; Giurgiutiu, V. Analytical and experimental investigation of the interaction of Lamb waves in a stiffened aluminum plate with a horizontal crack at the root of the stiffener. J. Sound Vib. 2018, 431, 212-225. [CrossRef]

29. Hamstad, M.A. Frequencies and amplitudes of AE signals in a plate as a function of source rise time. In Proceedings of the 29th European Conference on Acoustic Emission Testing, Vienna, Austria, 8-10 September 2010.

30. Gaudenzi, P.; Bernabei, M.; Dati, E.; De Angelis, G.; Marrone, M.; Lampani, L. On the evaluation of impact damage on composite materials by comparing different NDI techniques. Compos. Struct. 2014, 118, 257-266. [CrossRef]

31. Kumar, S.S.; Rao, K.S. Voice/non-voice detection using phase of zero frequency filtered speech signal. Speech Commun. 2016, 81, 90-103. [CrossRef]

32. Hu, C.; Wang, Z.; Zhu, Y.; Zhang, M. Accurate three-dimensional contouring error estimation and compensation scheme with zero-phase filter. Int. J. Mach. Tools Manuf. 2018, 128, 33-40. [CrossRef]

(C) 2020 by the authors. Licensee MDPI, Basel, Switzerland. This article is an open access article distributed under the terms and conditions of the Creative Commons Attribution (CC BY) license (http://creativecommons.org/licenses/by/4.0/). 